\title{
Radiopeptides for Imaging and Therapy: A Radiant Future
}

\author{
Kristell L.S. Chatalic ${ }^{1-3}$, Dik J. Kwekkeboom ${ }^{1}$, and Marion de Jong ${ }^{1,2}$ \\ ${ }^{1}$ Department of Nuclear Medicine, Erasmus MC, Rotterdam, The Netherlands; ${ }^{2}$ Department of Radiology, Erasmus MC, Rotterdam, \\ The Netherlands; and ${ }^{3}$ Department of Urology, Erasmus MC, Rotterdam, The Netherlands
}

Radiopeptides are powerful tools for diagnostic imaging and radionuclide therapy of various diseases. Since the introduction of the first radiopeptide into the clinical setting to diagnose neuroendocrine tumors about 25 y ago, many advances have been made in the field. This short review highlights novel strategies to improve the application of radiopeptides for imaging and therapy.

Key Words: radiopeptides; imaging; radionuclide therapy; developments; perspectives

J Nucl Med 2015; 56:1809-1812

DOI: 10.2967/jnumed.115.161158

Radiopeptides targeting somatostatin receptor (SSTR) have contributed significantly to patient care. The SSTR agonist octreotide and modifications thereof $\left(\left[\mathrm{Tyr}^{3}\right]\right.$ octreotide [TOC], [Tyr ${ }^{3}, \mathrm{Thr}^{8}$ ]octreotide [TATE], and [1-NaI ${ }^{3}$ octreotide [NOC]) are widely used in routine clinical practice for diagnosis of neuroendocrine tumors (NETs). ${ }^{111}$ In-diethylenetriaminepentaacetic acid-octreotide (OctreoScan; Mallinckrodt Inc.) was the first registered radiopeptide, and it has become the gold standard for diagnosis and staging of SSTR-positive tumors using SPECT and SPECT/CT. After the successful application of ${ }^{111}$ In-octreotide for imaging of NETs in the clinic, somatostatin analogs radiolabeled with $\beta$-emitting isotopes (i.e., ${ }^{177} \mathrm{Lu}$-DOTATATE and ${ }^{90}$ Y-DOTATOC) were introduced as treatment. Peptide receptor radionuclide therapy (PRRT) using ${ }^{177} \mathrm{Lu}$-DOTATATE and ${ }^{90}$ Y-DOTATOC has given impressive results thus far.

The success of radiolabeled somatostatin analogs inspired the scientific community to develop radiopeptides targeting different receptor families, including gastrin-releasing peptide (GRP), cholecystokinin-2/gastrin (CCK-2/CCK-B), glucagonlike peptide-1 (GLP-1), $\alpha_{\nu} \beta_{3}$-integrin, neurokinin-1 (NK-1), melanocytestimulating hormone (MSH), neuropeptide Y (NPY), luteinizing hormone-releasing hormone (LHRH), and chemokine receptor-4

Received Aug. 7, 2015; revision accepted Oct. 26, 2015.

For correspondence or reprints contact either of the following:

Marion de Jong, Erasmus MC, Department of Nuclear Medicine/Radiology,

Na-610, Postbus 2040, 3000 CA Rotterdam, The Netherlands.

E-mail: m.hendriks-dejong@erasmusmc.nl

Kristell L.S. Chatalic, Erasmus MC, Department of Nuclear Medicine/ Radiology/Urology, Na-620, Postbus 2040, 3000 CA Rotterdam, The Netherlands.

E-mail: k.chatalic@erasmusmc.nl

Published online Oct. 29, 2015.

COPYRIGHT (C) 2015 by the Society of Nuclear Medicine and Molecular Imaging, Inc.
(CXCR4) (1). Examples of radiopeptide families and their applications for tumor imaging and therapy are shown in Figure 1. Radiopeptides have established themselves as important agents for imaging, but also for PRRT. For medical imaging, peptides can be radiolabeled with radionuclides such as ${ }^{99 \mathrm{~m}} \mathrm{Tc},{ }^{111} \mathrm{In},{ }^{67} \mathrm{Ga}$, or ${ }^{123} \mathrm{I}$ for SPECT and ${ }^{18} \mathrm{~F},{ }^{68} \mathrm{Ga},{ }^{64} \mathrm{Cu},{ }^{86} \mathrm{Y}$, or ${ }^{124} \mathrm{I}$ for PET. For therapeutic purposes, peptides can be labeled with $\beta$-emitters such as ${ }^{177} \mathrm{Lu},{ }^{90} \mathrm{Y}$, or ${ }^{67} \mathrm{Cu}$ and $\alpha$-emitters such as ${ }^{213} \mathrm{Bi}$ or ${ }^{225}$ Ac. Current applications of clinical radiopeptides for imaging and therapy have been described in detail by Ambrosini et al. (2). Approaches intending to improve the performance of radiopeptides for imaging and therapy and thereby widen their scope of application are discussed below.

\section{NOVEL PERSPECTIVES FOR IMAGING}

Intensive research efforts have improved the pharmacokinetics and in vivo stability of radiopeptides, as well as boosting image quality by identifying radionuclides with more suitable nuclear physical properties or by combining nuclear imaging modalities with complementary modalities. Some examples of novel approaches for radionuclide therapy are shown in Figure 2.

\section{Change of Paradigm Using Receptor Antagonists}

An important eye-opener in the field of radiopeptides has been the recent introduction of SSTR antagonists, which showed more favorable pharmacokinetics and better tumor visualization than agonists did, despite their poor internalization rate. In a clinical study, the SSTR antagonist ${ }^{111}$ In-DOTA-BASS ( ${ }^{111}$ In-DOTA$\mathrm{pNO}_{2}$-Phe-c(DCys-Tyr-DTrp-Lys-Thr-Cys)DTyrNH ${ }_{2}$ ) resulted in higher tumor uptake and better visualization of metastatic NETs than did the SSTR agonist ${ }^{111} \mathrm{In}$-diethylenetriaminepentaacetic acidoctreotide (3).

Subsequently, radiolabeled GRPR-targeted antagonists also entered the arena, showing a more favorable pharmacokinetic profile and fewer side effects than agonists. GRPR-targeted antagonists have shown high potential in early clinical trials for prostate cancer imaging (4).

\section{Improving Pharmacokinetics and In Vivo Stability}

Many efforts have been made to increase the in vivo stability of radiopeptides by introducing structural modifications, although such modifications can cause undesired changes in pharmacokinetics or impaired receptor affinity. A remarkable advance in this area is the discovery of the in vivo enzyme inhibition concept, in which radiopeptides are administered together with an enzyme 


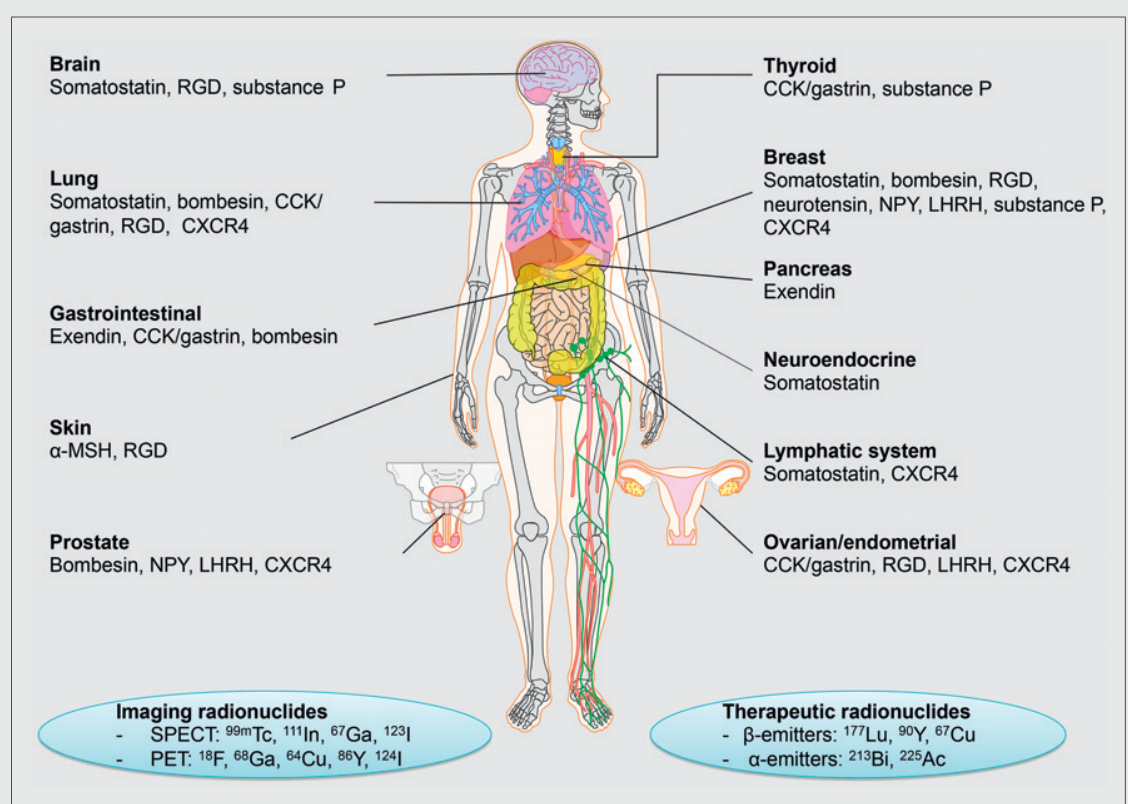

FIGURE 1. Examples of radiopeptide families, radionuclides, and their applications for imaging of tumors. RGD = arginylglycylaspartic acid.
For SPECT imaging, ${ }^{99 \mathrm{~m}} \mathrm{Tc}$ is the radioisotope of choice. However, recent developments in PET/CT and PET/MR technologies are steering the field toward positron emitters.

${ }^{68} \mathrm{Ga}$-radiopeptides are useful tools for PET/CT or PET/MRI of diseases, as ${ }^{68} \mathrm{Ga}$ can be obtained in-house from a ${ }^{68} \mathrm{Ge} /{ }^{68} \mathrm{Ga}$ generator. The introduction of ${ }^{68} \mathrm{Ga}$-labeled DOTA-conjugated somatostatin analogs (TOC, TATE, NOC) into the clinic, enabling PET/CT imaging of NETs, is an important advance in somatostatin-based imaging. The superior performance of imaging techniques using these tracers for detection of NET lesions was described by Ambrosini et al. (2).

Recently, ${ }^{68} \mathrm{Ga}$-labeled exendin- 4 has been introduced in the clinic for the detection of GLP-1 receptor-positive insulinomas (6). ${ }^{68} \mathrm{Ga}$-DOTA-exendin-4 PET/ CT is preferred over ${ }^{111}$ In-DOTA-exendin-4 SPECT/CT because of the higher spatial resinhibitor as a safeguard against enzymatic degradation. It has recently been shown that coinjection of a neutral endopeptidase inhibitor, such as phosphoramidon, can stabilize radiolabeled bombesin, minigastrin, and somatostatin analogs in vivo, leading to enhanced tumor uptake and improved tumor visualization (5). We believe that this strategy will have a significant impact on patient care, as it may enhance the diagnostic sensitivity and therapeutic efficacy of radiopeptides. For that purpose, a neutral endopeptidase inhibitor already applied in patients (e.g., thiorphan) is the preferred choice.

\section{${ }^{68} \mathrm{Ga}$ Versus ${ }^{18} \mathrm{~F}$}

Recently, imaging quality has been boosted by selecting radionuclides with more suitable nuclear physical properties.

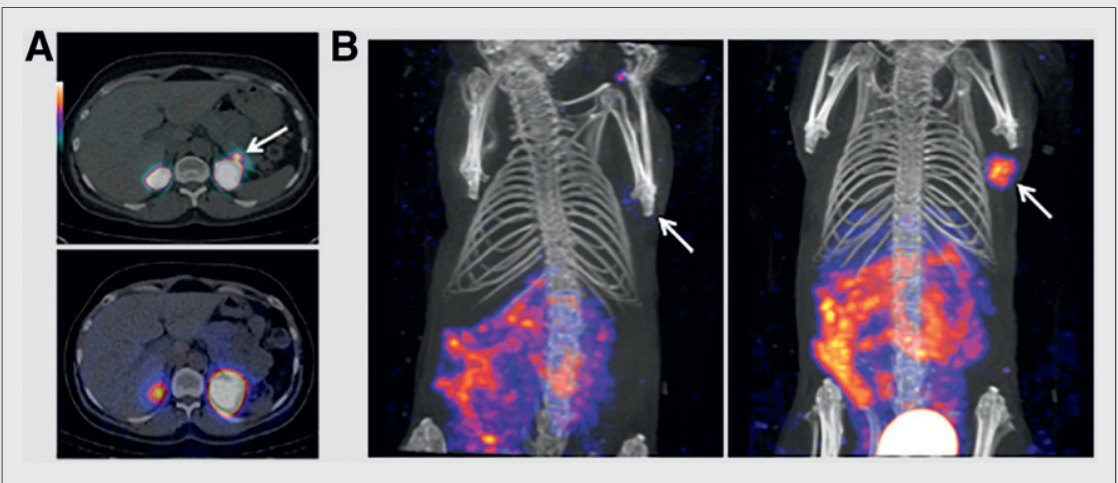

FIGURE 2. (A) PET/CT (top) of insulinoma patient showing focal ${ }^{6} \mathrm{Ga}-\mathrm{DOTA}$-exendin-4 uptake in distal pancreatic tail (consistent with surgically removed insulinoma) that was not shown by SPECT/CT (bottom) with ${ }^{111}$ In-DOTA-exendin-4. (B) SPECT/CT images of mice with GRPR-expressing PC-3 tumors (arrows) $4 \mathrm{~h}$ after injection of bombesin analog ${ }^{111}$ In-DOTA-PEG 2 -DTyr-GIn-Trp-Ala-Val- $\beta$ Ala-His-Phe-Nle- $\mathrm{NH}_{2}$ without (left) or with (right) coinjection of neutral endopeptidase inhibitor phosphoramidon $(300 \mu \mathrm{g})$. olution and better quantification options obtained with PET/CT cameras.

${ }^{18} \mathrm{~F}$-labeled peptides have some advantages over ${ }^{68} \mathrm{Ga}$-labeled peptides, such as a longer half-life. Moreover, the physical properties of ${ }^{18} \mathrm{~F}$ are more favorable for PET imaging than those of ${ }^{68} \mathrm{Ga}$. Indeed, an ${ }^{18} \mathrm{~F}$-labeled GRPR antagonist, BAY 864367 (3-cyano4- ${ }^{18} \mathrm{~F}$-fluorobenzoyl-Ala $\left(\mathrm{SO}_{3} \mathrm{H}\right)-\mathrm{Ala}\left(\mathrm{SO}_{3} \mathrm{H}\right)$-Ava-Gln-Trp-Ala-Val-

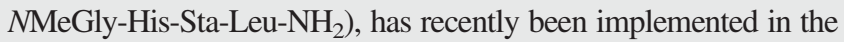
clinical setting (4). A drawback yet to be overcome with ${ }^{18} \mathrm{~F}$ labeled peptides is their higher lipophilicity, which gives rise to less favorable pharmacokinetics. An interesting approach consists of labeling peptides with $\mathrm{Al}^{18} \mathrm{~F}$ via radiometalation chemistry. Several peptides have been labeled with $\mathrm{Al}^{18} \mathrm{~F}$, such as octreotide, $\mathrm{E}[\mathrm{c}(\mathrm{RGDyK})]_{2}$, exendin-4, the bombesin analog NOTA-8-Aoc$\mathrm{BBN}(7-14) \mathrm{NH}_{2}$, and the GRPR antagonist JMV4168 (DOTA- $\beta$ Ala- $\beta$ Ala-JMV594) $(7,8)$. The development of a kit-based method for labeling peptides with ${ }^{18} \mathrm{~F}$ would make application of ${ }^{18} \mathrm{~F}$-labeled peptides possible in a wider range of hospitals.

\section{Nuclear/Optical Multimodality Imaging}

Nuclear imaging technologies available in the clinic (SPECT/CT, PET/CT, and PET/MR) are superior to any other clinical imaging modality in terms of specificity and sensitivity. However, optical imaging offers interesting applications as well and could complement the nuclear medicine uses (9). An important development in the field of radiopeptides 
has been the introduction of hybrid derivatives, containing both a fluorescent and a radioactive label, as these have significant implications in the field of imaging-guided surgery. Several hybrid radiopeptides have been developed, including agents targeting SSTR, GRPR, interleukin-11 receptor- $\alpha, \alpha_{\nu} \beta_{3}$-integrin, matrix metalloproteinase, CXCR4, and GLP-1R (10-12). Because of the size and lipophilicity of common fluorescent dyes, the main challenge lies in optimizing the chemical structures of hybrid tracers to preserve receptor affinity and biodistribution patterns while maximizing the range of tissue penetration.

\section{NOVEL PERSPECTIVES FOR THERAPY}

Novel strategies might improve the outcome of PRRT. These include the improvement of pharmacokinetics and in vivo stability, the combination of radionuclides, the combination of PRRT with chemotherapy, the use of $\alpha$-emitting radionuclides, and the use of novel methods to predict therapy response. Recent developments to increase the therapeutic effects of PRRT have been described in detail by Bison et al. (13). Some examples of novel approaches for radionuclide therapy are shown in Figure 3.

\section{Improving Pharmacokinetics and In Vivo Stability}

The success of PRRT would presumably be improved by enhancing the dose delivered to the tumor or limiting the dose delivered to normal healthy tissues. The use of SSTR antagonists has enhanced tumor targeting and prolonged tumor retention compared with SSTR agonists, resulting in an enhanced tumor radiation dose during PRRT. In a clinical study, the SSTR antagonist ${ }^{177}$ Lu-DOTA-JR11 exhibited higher tumor uptake and a longer intratumoral residence time than the SSTR agonist ${ }^{177}$ Lu-DOTATATE (14). This study was performed on only a few patients, and more systematic clinical studies are needed now to fully evaluate the role of ${ }^{177} \mathrm{Lu}-\mathrm{DOTA}-J R 11$ for therapy of NETs.

Patients with advanced prostate cancer could also benefit from PRRT, as treatment options for this patient group are limited. ${ }^{177} \mathrm{Lu}-$ GRPR antagonists have not been tested in clinical studies thus far, even though they have shown promising results in preclinical studies (15). Radionuclide therapy using PSMA-targeted tracers has been more widely explored, as PSMA expression has been validated in advanced prostate cancer in several reports. Extensive comparative studies showing target expression of PSMA and GRPR in advanced prostate cancer would guide the choice of therapeutic options for those patients.

\section{Combination Therapies}

In the search for increased therapeutic efficacy of PRRT, therapeutic radionuclides with different physical characteristics have been combined to target a wider range of tumor lesions. ${ }^{90} \mathrm{Y}$ and ${ }^{177} \mathrm{Lu} \beta$-emitting isotopes suitable for treatment of large and small tumors have been combined in simultaneous or sequential treatment. A recent retrospective study (16) described the benefits of the combination of ${ }^{90}$ Y-DOTATOC and ${ }^{177} \mathrm{Lu}$-DOTATOC over treatment with either radiopeptide alone, with increased survival

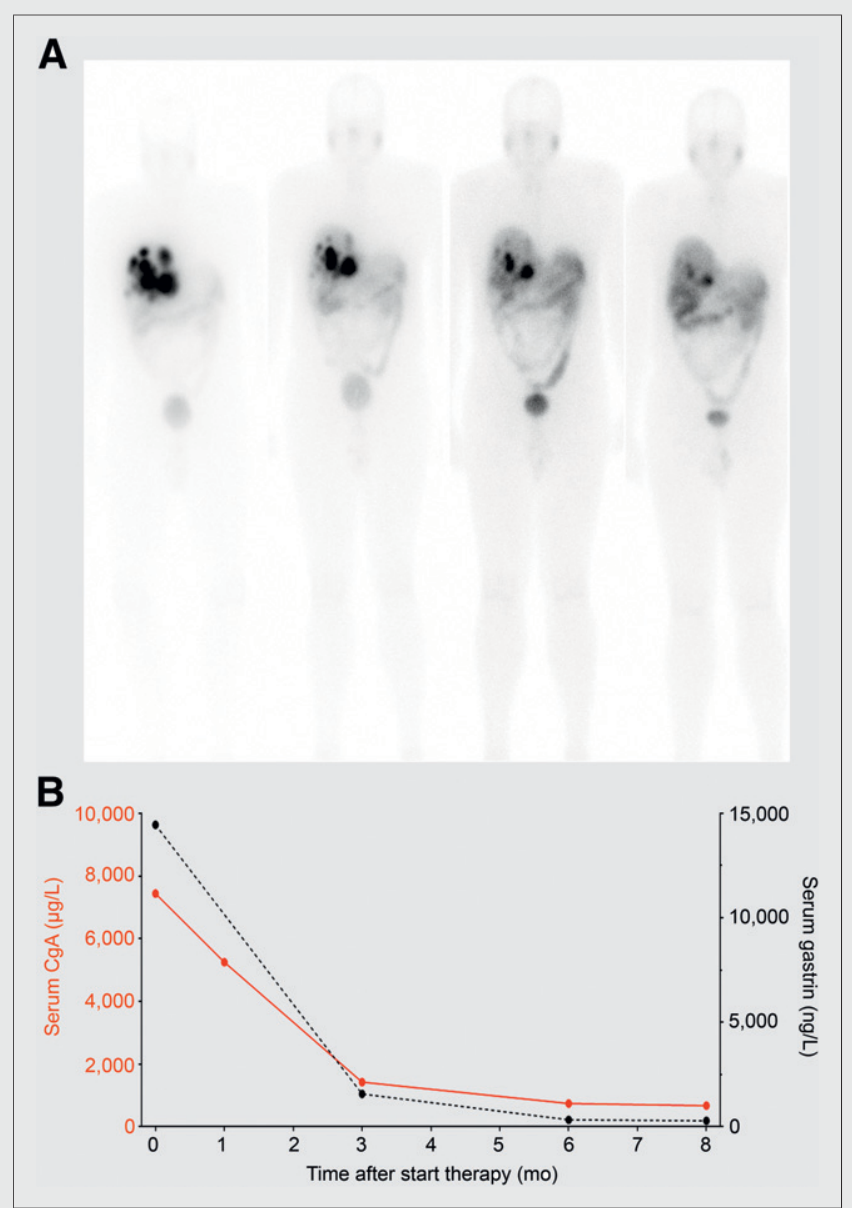

FIGURE 3. (A) Total-body images after each cycle of PRRT with $7.4 \mathrm{GBq}$ of ${ }^{177} \mathrm{Lu}$-octreotate in combination with low-dose capecitabine as chemosensitizer in patient with liver metastases of gastrinoma. Decreasing uptake in liver metastases indicates and preludes tumor response, which was later confirmed with $\mathrm{CT}$. (B) Course of tumor markers chromogranin- $\mathrm{A}(\mathrm{CgA})$ and gastrin.

after the combination treatment. However, patient selection bias cannot be excluded; therefore, prospective, randomized trials are needed before firm conclusions can be drawn.

PRRT can also be combined with other therapies to increase tumor response. Chemotherapeutics can sensitize tumor cells to PRRT by modulating DNA damage and repair mechanisms, apoptosis, cell proliferation, cell-cycle synchronization, and tumor cell oxygenation. However, careful design of such combination studies is needed, as chemotherapeutics can also alter receptor expression and tumor vascularization. For instance, the DNA alkylating drug temozolomide increased tumor perfusion in a preclinical study (13). Clinical studies combining PRRT using ${ }^{177} \mathrm{Lu}$-DOTATATE with several chemotherapeutics have been reported. Capecitabine and temozolomide both increased the response rate of ${ }^{177} \mathrm{Lu}$ DOTATATE in comparison with ${ }^{177}$ Lu-DOTATATE alone (17). A recent proof-of-concept study described the combination of PRRT with everolimus (18). It was shown that everolimus can safely be combined with ${ }^{177} \mathrm{Lu}$-octreotate to treat low-grade NETs. 


\section{Introduction of Powerful $\alpha$-Emitters}

An important development in the field of radionuclide therapy has been the introduction of $\alpha$-particle-emitting radionuclides. A first-in-human study showed that ${ }^{213} \mathrm{Bi}$-DOTATOC can eradicate neuroendocrine liver metastases pretreated with ${ }^{90} \mathrm{Y} /{ }^{177} \mathrm{Lu}-$ DOTATOC. Only moderate acute and midterm hematologic and renal toxicity was observed at effective therapeutic doses (19). Nonoperable and critically located gliomas have been treated with $\alpha$-targeted therapy, by local injection of ${ }^{213} \mathrm{Bi}$-DOTAsubstance $\mathrm{P}$, providing local irradiation of the tumor. The short tissue range of ${ }^{213} \mathrm{Bi}$ prevents damage to adjacent brain areas. Up till now, this therapy has proven to be feasible and safe, with only mild adverse effects observed (20). Despite the promising preliminary results obtained with ${ }^{213} \mathrm{Bi}$-DOTATOC and ${ }^{213} \mathrm{Bi}-$ substance $\mathrm{P}$ in patients, the short half-life of ${ }^{213} \mathrm{Bi}(45.6 \mathrm{~min}$ ) and the costs and limited availability of ${ }^{225} \mathrm{Ac} /{ }^{213} \mathrm{Bi}$ generators limit clinical use. The recently described accelerator-driven production of ${ }^{225} \mathrm{Ac}$ may allow it to be produced in quantities sufficient to supply centers for clinical trials and make the treatment available to a wider range of centers (21).

\section{Innovative Methods as Predictors of Therapeutic Response}

There is a clear need for personalization and standardization of PRRT to optimize efficacy and minimize long-term toxicity, as emphasized in a recent review of Bodei et al. (22). They recommended collecting data on several parameters to construct a patient-specific treatment plan, taking into account various patient-specific characteristics to predict the probability of response and predisposition to toxicity. Dosimetry can be used as a predictor of therapy response and toxicity; a relationship between tumor-absorbed dose and response has already been demonstrated for ${ }^{90} \mathrm{Y}$-DOTATOC (23).

Markers of DNA damage and repair, such as phosphorylated variant $\mathrm{H} 2 \mathrm{~A}$ histone family member $\mathrm{X}(\gamma-\mathrm{H} 2 \mathrm{AX})$, may predict tumor and normal-organ radiosensitivities. A recent paper described the use of a $\gamma-\mathrm{H} 2 \mathrm{AX}$-foci assay as a predictor of normal-tissue toxicity after PRRT (24). Genetic components can also predict disease stage and response to therapy (25).

\section{CONCLUSION}

Derived from natural peptide ligands, radiopeptides can be tailored and optimized to provide personalized treatment for several types of diseases. In recent years, several methods have been developed to optimize radiopeptides for imaging and therapy; these efforts and achievements may bridge the gap between the exciting promises of radiopeptides and their implementation in the clinic. Today, complex multimodal ligands are being designed for theranostic applications, whereas simple radiopeptides combine all the key ingredients for effective theranostic applications, including easy manufacturing, fast clearance, low immunogenicity, and efficient targeting. We believe that the recent advances described in this article may lead the way to a radiant future for radiopeptides in the field of theranostics.

\section{DISCLOSURE}

Marion de Jong and Dik Kwekkeboom own stock in Advanced Accelerator Applications (AAA). No other potential conflict of interest relevant to this article was reported.

\section{REFERENCES}

1. Koopmans KP, Glaudemans AW. Rationale for the use of radiolabelled peptides in diagnosis and therapy. Eur J Nucl Med Mol Imaging. 2012;39(suppl 1):S4-S10.

2. Ambrosini V, Fani M, Fanti S, Forrer F, Maecke HR. Radiopeptide imaging and therapy in Europe. J Nucl Med. 2011;52(suppl 2):42S-55S.

3. Wild D, Fani M, Behe M, et al. First clinical evidence that imaging with somatostatin receptor antagonists is feasible. $J$ Nucl Med. 2011;52:1412-1417.

4. Sah BR, Burger IA, Schibli R, et al. Dosimetry and first clinical evaluation of the new ${ }^{18} \mathrm{~F}$-radiolabeled bombesin analogue BAY 864367 in patients with prostate cancer. J Nucl Med. 2015;56:372-378.

5. Nock BA, Maina T, Krenning EP, de Jong M. "To serve and protect": enzyme inhibitors as radiopeptide escorts promote tumor targeting. J Nucl Med. 2014;55:121-127.

6. Antwi K, Fani M, Nicolas G, et al. Localization of hidden insulinomas with ${ }^{68}$ Ga-DOTA-exendin-4 PET/CT: a pilot study. J Nucl Med. 2015;56:1075-1078.

7. Laverman P, McBride WJ, Sharkey RM, Goldenberg DM, Al Boerman OC. ${ }^{18} \mathrm{~F}$ labeling of peptides and proteins. J Labelled Comp Radiopharm. 2014;57:219-223.

8. Chatalic KL, Franssen GM, van Weerden WM, et al. Preclinical comparison of Al18Fand ${ }^{68} \mathrm{Ga}$-labeled gastrin-releasing peptide receptor antagonists for PET imaging of prostate cancer. J Nucl Med. 2014;55:2050-2056.

9. van Leeuwen FW, de Jong M. Molecular imaging: the emerging role of optical imaging in nuclear medicine. Eur J Nucl Med Mol Imaging. 2014;41:2150-2153.

10. Kuil J, Velders AH, van Leeuwen FW. Multimodal tumor-targeting peptides functionalized with both a radio- and a fluorescent label. Bioconjug Chem. 2010;21:1709-1719.

11. Azhdarinia A, Ghosh P, Ghosh S, Wilganowski N, Sevick-Muraca EM. Dual-labeling strategies for nuclear and fluorescence molecular imaging: a review and analysis. $\mathrm{Mol}$ Imaging Biol. 2012;14:261-276.

12. Brand C, Abdel-Atti D, Zhang Y, et al. In vivo imaging of GLP-1R with a targeted bimodal PET/fluorescence imaging agent. Bioconjug Chem. 2014;25:1323-1330.

13. Bison SM, Konijnenberg MW, Melis M, et al. Peptide receptor radionuclide therapy using radiolabeled somatostatin analogs: focus on future developments. Clin Transl Imaging. 2014;2:55-66.

14. Wild D, Fani M, Fischer R, et al. Comparison of somatostatin receptor agonist and antagonist for peptide receptor radionuclide therapy: a pilot study. J Nucl Med. 2014;55:1248-1252

15. Dumont RA, Tamma M, Braun F, et al. Targeted radiotherapy of prostate cancer with a gastrin-releasing peptide receptor antagonist is effective as monotherapy and in combination with rapamycin. J Nucl Med. 2013;54:762-769.

16. Radojewski P, Dumont R, Marincek N, et al. Towards tailored radiopeptide therapy. Eur J Nucl Med Mol Imaging. 2015;42:1231-1237.

17. Claringbold PG, Turner JH. Pancreatic neuroendocrine tumor control: durable objective response to combination Lu-octreotate-capecitabine-temozolomide radiopeptide chemotherapy. Neuroendocrinology. June 10, 2015 [Epub ahead of print].

18. Claringbold PG, Turner JH. Neuroendocrine tumor therapy with lutetium-177octreotate and everolimus (NETTLE): a phase I study. Cancer Biother Radiopharm. 2015;30:261-269.

19. Kratochwil C, Giesel FL, Bruchertseifer F, et al. ${ }^{213}$ Bi-DOTATOC receptor-targeted alpha-radionuclide therapy induces remission in neuroendocrine tumours refractory to beta radiation: a first-in-human experience. Eur J Nucl Med Mol Imaging. 2014;41: 2106-2119.

20. Krolicki L, Morgenstern A, Kunikowska J, et al. Alphaknife for glioma tumors: two years experiences with targeted alpha therapy of ${ }^{213} \mathrm{Bi}$-substance P [abstract]. Eur $J$ Nucl Med Mol Imaging. 2014;41(suppl 2):S291-S292.

21. Weidner JW, Mashnik SG, John KD, et al. ${ }^{225} \mathrm{Ac}$ and ${ }^{223} \mathrm{Ra}$ production via $800 \mathrm{MeV}$ proton irradiation of natural thorium targets. Appl Radiat Isot. 2012;70:2590-2595.

22. Bodei L, Kidd M, Baum RP, Modlin IM. PRRT: defining the paradigm shift to achieve standardization and individualization. J Nucl Med. 2014;55:1753-1756.

23. Strigari L, Konijnenberg M, Chiesa C, et al. The evidence base for the use of internal dosimetry in the clinical practice of molecular radiotherapy. Eur J Nucl Med Mol Imaging. 2014;41:1976-1988.

24. Denoyer D, Lobachevsky P, Jackson P, Thompson M, Martin OA, Hicks RJ. Analysis of ${ }^{177} \mathrm{Lu}$-DOTA-octreotate therapy-induced DNA damage in peripheral blood lymphocytes of patients with neuroendocrine tumors. $J$ Nucl Med. 2015;56:505-511.

25. Modlin IM, Oberg K, Taylor A, Drozdov I, Bodei L, Kidd M. Neuroendocrine tumor biomarkers: current status and perspectives. Neuroendocrinology. 2014; 100:265-277. 